\title{
Malicious Pixels-Using QR Codes in Dentistry-A Review
}

Srinivasan $\mathrm{K}^{1}$, Chitra $\mathrm{S}^{2}$

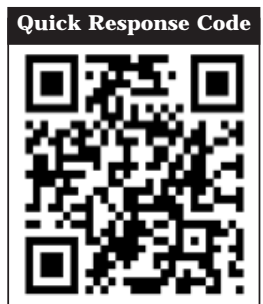

doi: $10.5866 / 2017.9 .10092$

${ }^{1 \& 2}$ Private Practitioner,

1/24, Kamaraj Street,

VOC Nagar, Sankaranpalayam,

Vellore (TN State)

\section{Article Info:}

Received: April 8, 2017

Review Completed: May 7, 2017

Accepted: J une 9, 2017

Available Online: J une, 2017 (www.nacd.in)

(c) NAD, 2017 - All rights reserved

Email for correspondence:

skskskpedo@gmail.com

\section{ABSTRACT:}

Quick response (QR) codes are two-dimensional barcodes that are used to encode and decode information. QR code is a popular form of barcode pattern that is ubiquitously used to tag information to products or for linking advertisements. QR codes have become popular for allowing smart phones and other camera-equipped, web-connected devices access to web sites, text messages, and application downloads. QR codes are read by a mobile device through the device's camera using an application specifically designed to read the information embedded in the code. QR codes are easily recognizable by their square shape and series of black square/white space patterns. The size of the QR code is largely irrelevant: as long as the device's camera can bring the code into the field of view, the code can be read by the code reader application.

QR code is an innovative approach in which patient details and radiographic interpretation can beencoded. These QR codes can be shared efficiently worldwide with easy authentication. QR code is a boost to early diagnosis and retrospective studies. Implementing QR code in Dentistry will save time eliminate paper work, and problems encountered in storage of patient details with radiographic interpretation and minimize manual efforts in retrieval of information. The present article suggests potential uses of QR codes in Dentistry with an aim to motivate the fraternity to be innovative and use this tool for improving quality of health care.

Key words: Barcodes, Dentistry, QR codes, Quick Response Codes, Quick response scanner, Smartphones.

\section{INTRODUCTION}

QR code is a Matrix code. In the year 1994 brainchild of Denso Wave, a subsidiary of the J apanese carmaker, Toyota, QR codes were originally designed, for tracking parts in automobile manufacturing, but they have been an integral part of the J apanese consumer landscape for more than a decade. ${ }^{1}$ This Matrix code technology has been around for over a decade but has since become popular as a medium for marketers to reach smart phone users. Quick Response codes, or QR codes, are nothing new. In fact, in J apan and E urope they have been used in marketing as well as inventory control and manufacturing for thelast 10 years. The security of one-dimensional (1D) barcodes is lower than 2D barcodes. 1D barcodes are very easy to read 
by scanning the lines and the spaces. However, 2D barcodes are not easy to read a symbol pattern by human eyes. With regard to readability, 1D barcodes must scan along a single directional. If the angle of a scan line does not fit within a range, the data would not be read correctly. However, 2D barcodes get wide ranges of angles for scanning. Thus, 2D barcodes are readability. ${ }^{2}$

The key difference between the two is the amount of data they can hold or share. Bar codes are linear one-dimensional codes and can only hold up to 20 numerical digits, whereas QR codes are two-dimensional (2D) matrix barcodes that can hold 7,089 numeric characters and 4,296 al phanumeric characters, and 1,817 kanji characters of information. ${ }^{1}$

A QR code has the ability to hold more information and their ease of use makes them practical for small businesses. When a person scan or read a QR code with your i Phone, Android or other camera enabled Smartphone, we can link to digital content on the web; activate a number of phone functions including e-mail, IM and SMS; and connect the mobile device to a web browser. Any of these desired functions are easily achieved by properly creating the QR code. It's a simple process of entering the appropriate data into the QR code generators. ${ }^{3}$

It can be read by the camera of a Smartphone, and once read it may instantly redirect the Smartphone user to a webpage. QR codes are only to be machine readable. This means that a human looking at the code is unable to determine its content. QR codes can be used in a variety of ways to market a business, to provide further information on a product or service by encoding general text, URL, phone number, business card and even provide Wi$\mathrm{Fi}$ access. ${ }^{3}$ In the Philippines, the National Bureau of Investigation uses QR codes in their NBI clearances. ${ }^{4}$

A recent implementation of QR codes is India's 'Aadhaar' project that gives a unique identification number to the citizens of I ndia much like the Social Security Number (SSN) in USA. Another first in Indian aviation (J et Airways) uses QR codes are in their products and services.

\section{QR codes $^{5}$}

There are 40 versions (sizes) of QR codes that consist of different areas that are reserved for specific purposes. In the following version 2 of QR codes are coated (Figure 1), because version 1 does not contain all areas.



Figure 1: Structure of QR code Version 2

a) Finder Pattern (1): The finder pattern consists of three identical structures that are located in all corners of the QR code except from the bottom right one. Each pattern is based on a $3 \times 3$ matrix of black modules surrounded by white modules that are again surrounded by black modules. The Finder Patterns enable the decoder software to recognize the QR code and determine the correct orientation.

b) Separators (2): The white separators have a width of one pixel and improve the recognizability of the Finder Patters as they separate them from the actual data.

c) Timing Pattern (3): Alternating black and white modules in the Timing Pattern enable the decoder software to determine the width of a single module.

d) Alignment Patterns (4): Alignment Patterns support the decoder software in compensating for moderate image distortions. Version $1 \mathrm{QR}$ codes do not have Alignment Patterns. With growing size of the code, more Alignment Patterns are added.

e) Format Information (5): The Formation Information section consists of 15 bits next to the separators and stores information about the error correction level of the QR code and the chosen masking pattern.

f) Data (6): Data is converted into a bit stream and then stored in 8 bit parts (called code words) in the data section. 
g) Error Correction (7): Similar to data codes, error correction codes are stored in 8 bit long code words in the error correction section.

h) Remainder Bits (8): This section consists of empty bits of data and error correction bits cannot be divided into 8 bit code words without remainder.

The entire QR code has to be surrounded by the so-called Quiet Zone, an area in the same col or shade as white modules, to improve code recognition by the decoder software

Quick response codes has the ability to handle different types of data such as numeric and al phabetic characters, Kanji, Hiragana, Katakana, symbols, binary and control codes. QR code has the advantage of smaller printout size, high speed scanning, allows for artistic representations (QR code art) and can be recognized correctly even if $50 \%$ areas of barcode are damaged. ${ }^{7} \mathrm{QR}$ codes can be read by freely available software using any smart phone, tablet or laptop with a camera, or by a QR code scanner. ${ }^{8,9}$

\section{QR codes data types ${ }^{4}$}

QR codes can contain many different types of information. Different app readers on Smartphone are able to act and read this data. Think of it as an alternative way of getting data into your phone (as opposed to typing it in manually). Here are some of the possibilities.

a) Contact information: QR codes can contain contact information so that someone can easily scan a QR code, view their contact details, and add you on their phone. Other inputs likename, phone number, e-mail, address, website, memo, and more can be incorporated.

b) Calendar event: If an event want to promoted, a QR code containing info for that event to be created which can contain event title, start and end date/time, time zone, location, and description. This could work well on an event flyer or possibly even on a website promoting.

c) E-mail address: A QR code can contain your email address so that someone can scan the code, see your e-mail, and then open an e-mail on their phones. If your call to action is mostly to have someone e-mail you, this would-be great.

d) Phonenumber: If e-mail isn't immediateenough and you want someone to call then link them up to a phone number.

e) Geo location: QR code linking someone to a Google Maps location. This will allow someone to scan QR code and get directions so they don't have to manually type in an address. Although some may prefer to type it in.

f) SMS: QR codes can populate a text message with a number and message. A person can have their QR code and send a text saying "Tell me more about $X Y Z$," for instance.

g) Text: A sentence or a paragraph of text can in the QR codeformat. This could befun for having some type of QR code based game where a person can leave hints in QR codes.

h) Wi-Fi network: WEP wireless key can be set upto that someone can scan a QR code and automatically configure Wi-Fi on their phones.

i) URL: The possibilities of encoding URL into barcode are endless. A link that takes someone to your Face book fan page, Linkedl $n$ or Twitter profile, YouTube video Link, Check in to some place via check in link, pay for something via PayPal.

Encoding android market or i Phone app store link allows promoting and downloading the mobile applications anywhere. Some of the QR codegenerating sites are Qurify, Kaywa, Scan buy, QR Stuff and Delivr. Google's URL Shortener can also create a QR code from a shortened link. For mobile phone users, I P hones offer a downl oadable app from the iTunes store. BlackBerry, Android and other mobile phone platform users can download an app from Scan Life.

\section{Applications QR codes}

QR codes were developed for the automotive industry but have found many applications in various fields. With the integration of technology and healthcare on much front sand the majority of urban global population having access to mobile devices \& smart phones; decoding a QR code to access valuable information is not unthinkable.

\section{Applications QR codes in Medicine}

QR Codes are being used for patient identification by printing it on patient wrist band, which encodes for information like patient's name, identification number, date of birth, sex, ward and bed numbers. ${ }^{10}$ QR Codes are given by 
Physiotherapists to the patient for postoperative physical exercises which provides access to multimedia sources on the web that contain video demonstrations of the exercises, or some additional suggestions for a good recovery. ${ }^{11} \mathrm{QR}$ Codes are used in blood test process management by marking the test-tube with it and are inserted into the tester. The system automatically examines the inserted blood. ${ }^{12}$

\section{Applications QR codes in Dentistry}

QR codes can have a wide range of application in various aspects of Dentistry. They can be used as a tool in Dental education, product promotion and practice management.

Dental Education: QR Codes can be used to maintain case records, to code radiographs, to print codes on IOPA covers which encode case history and radiographic interpretation. ${ }^{\mathbf{1 3 - 1 7}} \mathrm{QR}$ Codes will be useful to maintain community Dentistry case records and in follow up record maintenance. In Prosthodontics, QR Codes can be used for recognition of casts and dentures. Tooth eruption patterns and tooth movement details can be updated using QR Codes.

QR Codes will be useful in setting up a digital museum.

Dental material composition information \& methods of material manipulation, stepwise guidance to procedures such as impression making and cavity preparation can be encoded in QR Codes which will help in Undergraduate teaching in institutions.

In the field of Dental education QR codes can revolutionize the accessibility that students will have to scientific material available on the web.

- In the Undergraduate and Post graduate teaching Institution QR code helps in learning process as it is of help by using it for spot diagnosis of casts and developmental anomalies.

They also can be used to give students brief overview of a topic just by scanning a QR code before entering a lecture, workshops or seminars. They can be used in lecture notes or presentations to get access to detailed information from an elearning site or specific scientific material available on the web. Each lecture slide can have different QR codes for video links, journal access or learning information relevant to the topic.
They can be used to code standard operating procedures, give instruments lists for specific cl inical procedures and to conduct student surveys. These codes can be used for self assessment exams and OSCE training sessions where the answers to the questions can be coded to maintain secrecy and decrease the workload on the academicians guiding the session.

QR codes can be used to give accessibility to students to online surveys, faculty feedback and course evaluation forms. The student's progress/ assignments or projects can be reviewed.

Easy access to student digital portfolios for regular updates, each student can be assigned a QR code at the beginning of the year or start of their course.

Among other uses, information encrypted on a QR code can help in orientation of a new student through the Dental school/campus by providing maps, videos and layouts of the school with information regarding the departments and clinics or labs.

QR codes on the casts and dentures help in easy recognition.

In Orthodontics tooth movement details can be updated instead of maintaining the case report that takes a longer time.

\section{Product Promotion ${ }^{13-17}$}

QR codes can be used to deliver detailed information on Dental products such as toothbrushes, Dental floss, mouthwashes, advanced oral hygiene aids as well as Oral Cancer detection kits and saliva testing kits. The information provided on product leaflets could be enriched and elaborated by using the QR codes, which would help in patient education and motivation. Thus leading to better compliance towards usage of Dental products.

\section{Practice Management ${ }^{13-17}$}

In the field of practice management these codes could be used to find directions to Dental schools/ Dental clinics, advertisements for new treatment procedures, video links to Dental surgical procedures, postoperative instructions, patient awareness and wellness data.

Patients seated in waiting areas could use these codes to access general health information, get 
information regarding various biomaterials used in Dentistry, profile information of attending Dentists and Specialists in a clinic, post testimonials online and use the link to respond to patient assessment questionnaires.

QR Code on a brochure can facilitate initial patient doctor contact by linking them to a website to get more information, or book an appointment. It is indeed a simple and effective way to improve the image of an organization on a technical, user friendly and eco-friendly front.

Dental professionals can access current clinical information and decision support at the point of care by ease in access to online medical and dental text books, professional guidelines, drug references and evidence based reviews.

They may also aid the health care provider to allay the apprehensions of patients instantly by showing them videos of Dental procedures and other materials.

In Dentistry QR code can be used as procedure reminder in clinic for infrequently performed procedures, such as checking the oxygen tank, starting up /shutting down the X-ray processor, etc.

QR code linked to a short video can be helpful in describing patient selection criteria, tips for successful completion of the competency, and common errors.

It can also be used in the Dental assisting clinic for tray setup guide, and on surgical instruments to identify devices in order for better management and to contribute to patient safety.

The emerging trend of QR code has gained interest in different fields of Dentistry an innovative approach to record Radiographs details using QR code. The approach enables to have both case history and radiographic interpretation coded and scanned on the radiograph cover, which can be read using a QR scanner or with a mobile having QR codescanner software.

It provides an easy way in maintaining case records and biopsy reports in histopathology.

The working of an electronic Dental chair, the Dental material composition information and methods of material manipulation, step-wise guidance to procedures such as impression making and cavity preparation can be decoded by QR code in Clinical Dentistry.
In Dentistry, QR Codes are used in the Dental assisting clinic for tray setup guide for procedures like minor surgeries like impaction, flap surgery and RCT.

QR Codes present on surgical instruments are used to identify devices in order for better management and to contribute to patient safety.

Forensic Dentistry is finding increased application in identifying deceased individuals.

A new denture identification system using Quick Response Codeincorporated in dentureswhich are less cost-effective, store lot of patient-related information and be easily accessible to the Dentist.

\section{Merits $^{16-17}$}

QR code also has the advantage of being inexpensive and readable from any form of display e.g. paper prints, digital displays, banners, stickers etc. temporarily added to campus signs for a class activity. In academic uses, QR codes on student tests could help ensure anonymity in grading.

QR codes might lead students to open forums where they could join in community discussions about what they've heard or seen. In scientific endeavors, QR codes could take the place of printed labels; attached to lab work, samples, or medication options, they could preserve confidentiality of participant names. The approach using QR code enables to have both case history and biopsy report coded and scanned on either side of the slide and facilitates retrospective analyses for the Pathol ogist. It also helps the patient by providing easy access to the biopsy report through mobile using QR Code scanner. It decreases paper work and reduces manual effort in maintaining and retrieval of the histo-pathological records. Other merits are no seepage of monomer into the QR Code, Simple to construct, no distortion or air voids over the QR Code, gives definite information.

\section{Demerits ${ }^{16-17}$}

Lack of familiarity of Quick Response Codes and smart phones with QR Codescanner softwarelimits the usage of $Q R$ code. Incorporation of histopathological pictures in the QR Code is not possible which can be done with the advanced versions in the future. Several researchers believe that the privacy and confidentiality are at stake using QR Code. This can be overcome using Security QR Code (SQRC) which provides password protection for the QR Codes. 


\section{Future I mplications}

As QR codes become more popular and students and faculty, both begin using them with more frequency, more strategies for implementing QR code technology with ubiquitous mobile access devices will become apparent.

\section{Conclusion}

QR code, a new approach in histopathology, should be practiced in order to facilitate long term retrospective studies which saves a lot of time, by electronically recording the details, and provides ease of accessibility from anywhere in the world through mobiles, maintaining confidentiality of report information. Several offline and online software are advanced at aiming rapid progress in retrospective research in order to decode diagnostic dilemmas. I nnovatively translating modern oral pathological practice is the need of the hour.

\section{References:}

1. QR code.com [home page on internet]. J apan: Densowave Incorporated [Last accessed on $2012 \mathrm{Feb} 20$ ]. Available from: http://www.denso-wave.com/qrcode/indexe.html .

2. J un Chou Chuang, Yu Chen $\mathrm{Hu} \&$ Hsien J u Ko. A Novel Secret Sharing Technique Using QR Code. Int J I mage Processing 2010; 4(5):468-75.

3. J eff Korhan. How QR Codes Can Grow Your Business, 2011. [Available]: http://www.socialmediaexaminer.com/howqrcodes-can-grow-your-business/.

4. Sankara Narayanan. QR Codes and Security Solutions. Int J Computer Sci Telecommunications 2012; 3(7).

5. Gao J Z, Prakash L, and J agatesan R. Understanding 2dbarcode technol ogy and applications in $\mathrm{m}$-commerce- design and implementation of a $2 \mathrm{~d}$ barcode processing solution. In COMPSAC2007; (2):49-56.

6. Diazgranados M, Funk VA. Utility of QR codes in biological collections. Phyto Keys 2013; 21-34.

7. Sahu SK, Gonnade SK. QR code and application in India. Int J RecentTechnol Eng 2013; 2:26-8.

8. Sharma V. A study of malicious QR codes. Int J Comput Intell Inf Secur 2012; 3:12-7.

9. Chuang $\mathrm{C}, \mathrm{Hu} Y \mathrm{C}, \mathrm{KoHJ}$. A Novel secret sharing technique using QR code. Int J I mage Process 2010; 4:468-75.

10. Soon TJ. The QR Code - Section three. The Synthesis J ournal 2008:59-78.

11. Kubben LP. QR codes in neurosurgery. Surg Neurol Int 2011; 2:104.

12. Garcia Betances RI, Huerta MK. A review of automatic patient identification options for public healthcare centers with restricted budgets. OnlineJ Public Health I nformatics 2012; 4(1):1-16.

13. Corina Laura Stefanescul, Marius Florentin Popa, LaviniaSimona Candea, I onut Parlica. Study on forensic dental identification methods by labeling prosthetic restorations. Romanian Soc Legal Medicine 2015; 23:37-42.

14. Ashish R J ain, P Sindhu, CJ Venkata Krishnan, Chitraa R Chandran. A New Alternative Technique for Denture Identification case report. World J Dent 2015; 6(3):188-92.

15. Moidin Shakil, Durbakula Karteek, Kulkarni Spoorti, Maji J ose. Quick response code in Oral and Maxillofacial Radiology. J Oral Maxillofacial Rad 2014; 2(3).

16. Moidin Shakil, Karteek Durbakula, Kulkarni Spoorti, Maji J ose. Yen code-it's Application In Dentistry. J Mobile Technology Med 2015; 4(2).

17. Telang A, Chakravarthy PVK, Lahari AT, Nerali J T. QR Codes in Dentistry. Malaysian Dent J 2012; 34(2).

\section{Gain quick access to our journal online View our journal at www.nacd.in}

